\title{
Erratum to: Variants of the Selberg sieve, and bounded intervals containing many primes
}

DHJ Polymath*

*Correspondence:

tao@math.ucla.edu Department of Mathematics, University of California, LoS Angeles, Los Angeles, CA, USA

\section{Erratum to: Research in the Mathematical Sciences (2014) 1:12 DOI: 10.1186/s40687-014-0012-7}

The arXiv identifiers for some of the references should be added for completeness. These references are:

9. Banks, W.D., Freiberg, T., Maynard, J.: On limit points of the sequence of normalized prime gaps. http://arxiv.org/abs/1404.5094.

10. Banks, W.D., Freiberg, T., Turnage-Butterbaugh, C.L.: Consecutive primes in tuples. http://arxiv.org/abs/1311.7003.

11. Benatar, J.: The existence of small prime gaps in subsets of the integers. http://arxiv. org/abs/1305.0348.

12. Castillo, A., Hall, C., Lemke Oliver, R.J., Pollack, P., Thompson, L.: Bounded gaps between primes in number fields and function fields. http://arxiv.org/abs/1403.5808.

13. Chua, L., Park, S., Smith, G.D.: Bounded gaps between primes in special sequences. http://arxiv.org/abs/1407.1747.

14. Freiberg, T.: A note on the theorem of Maynard and Tao. http://arxiv.org/ abs/1311.5319.

15. Li, H., Pan, H.: Bounded gaps between primes of the special form. http://arxiv.org/ abs/1403.4527.

16. Maynard, J.: Dense clusters of primes in subsets. http://arxiv.org/abs/1405.2593.

17. Pintz, J.: On the ratio of consecutive gaps between primes. http://arxiv.org/ abs/1406.2658.

18. Pintz, J.: On the distribution of gaps between consecutive primes. http://arxiv.org/ abs/1407.2213.

19. Pollack, P.: Bounded gaps between primes with a given primitive root. http://arxiv. org/abs/1404.4007.

20. Pollack, P., Thompson, L.: Arithmetic functions at consecutive shifted primes. http://arxiv.org/abs/1405.4444.

() 2015 Polymath. 
21. Thorner, J.: Bounded gaps between primes in Chebotarev sets. http://arxiv.org/ abs/1401.6677.

35. Pintz, J.: Polignac numbers, conjectures of erdos on gaps between primes, arithmetic progressions in primes and the bounded gap conjecture. http://arxiv.org/ abs/1305.6289.

Note: 21 has now appeared in Research in the Mathematical Sciences 2014, 1:4.

The online version of the original article can be found under doi:10.1186/s40687-014-0012-7.

Published online: 30 July 2015

Submit your manuscript to a SpringerOpen ${ }^{\circ}$ journal and benefit from:

- Convenient online submission

- Rigorous peer review

- Immediate publication on acceptance

- Open access: articles freely available online

- High visibility within the field

- Retaining the copyright to your article

Submit your next manuscript at $\boldsymbol{s p r i n g e r o p e n . c o m ~}$ 